\title{
Association between Intracellular Infectious Agents and Schizophrenia
}

\author{
Mi-Hee Park', Young-Joon Kwon', Hee-Yeun Jeong1, Hwa-Young Lee', Young Hwangbo ${ }^{1}$, Hee-Jung Yoon ${ }^{3}$, \\ Se-Hoon Shim ${ }^{1}$ \\ Departments of ${ }^{1}$ Psychiatry and ${ }^{2}$ Preventive Medicine, Soon Chun Hyang University Cheonan Hospital, Soon Chun Hyang University \\ College of Medicine, Cheonan, ${ }^{3}$ Division of Infectious Diseases, Department of Internal Medicine, Eulji University Graduate School, Daejeon, \\ Korea
}

\begin{abstract}
Objective: A number of studies have reported association between Toxoplasma gondii (T. gondii) and Chlamydia infection and the risk of schizophrenia. The aim of the present study was to compare the prevalence of $T$. gondii and Chlamydia infection between the schizophrenia and normal control subjects and to compare the clinical features between seropositive and seronegative schizophrenia patients.

Methods: The rate of serum reactivity to T. gondii, Chlamydia trachomatis ( $C$. trachomatis), Chlamydia pneumonia in 96 schizophrenia and 50 control subjects was investigated using enzyme-linked immunosorbent assay and indirect fluorescent antibody technique. The clinical symptoms of the schizophrenia patients were scored with Positive and Negative Syndrome Scale and a comparative analysis was carried out.

Results: A significant positive association between immunoglobulin $\mathrm{G}(\mathrm{IgG})$ antibodies to $T$. gondii and $C$. trachomatis in schizophrenia was found, and the odds ratio of schizophrenia associated with IgG antibody was found to be 3.22 and 2.86, respectively. The Toxoplasma-seropositive schizophrenia patient had higher score on the negative subscale N1 and N7 and general psychopathology subscale G13, while C. trachomatis-seropositive schizophrenia patient had higher score on the general psychopathology subscale G10.

Conclusion: The results from the present study suggest significant association between T. gondii, C. trachomatis infection and schizophrenia. In future, further studies are needed to elucidate the correlation between the two types of infection and schizophrenia.
\end{abstract}

KEY WORDS: Schizophrenia; Toxoplasma; Chlamydia trachomatis; Chlamydia pneumoniae.

\section{INTRODUCTION}

Several causative factors have been identified in the pathophysiology of schizophrenia. Family and twin studies indicate that there is a strong genetic component, which influences the risk of acquiring schizophrenia. However, epidemiological and neuropathological studies have also indicated that some cases of schizophrenia may be associated with environmental factors, such as birth season or exposure to infectious agents. ${ }^{1,2}$

Until now, various microbial agents have been proposed as risk factors for schizophrenia, and they all share

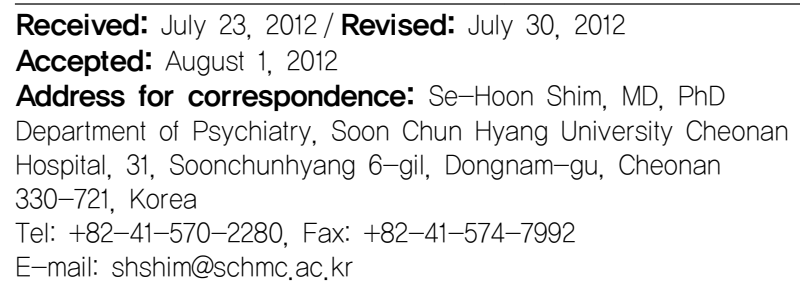

similar characteristics; an ability to establish persistent infections within the central nervous system as well as the occurrence of psychiatric symptoms in some individuals infected with these agents. We found several published studies from various research groups with special emphasis on the protozoan organism Toxoplasma (T.) gon$\mathrm{dii}^{3)}$ as well as intracellular bacteria Chlamydia.

T. gondii is a neurotropic protozoan parasite that was characterized at the turn of the 20th century and later associated with a series of congenital sensory and neurological conditions. ${ }^{4)}$ Although the definitive hosts are felines such as cats, T. gondii affects almost all warm-blooded animals including humans. In humans, infection is mainly acquired by ingestion of contaminated food or water or through eating undercooked or raw meat that contains tissue cysts. ${ }^{5)}$ This microbe is known to be neurotrophic and infects both neurons and glia. ${ }^{6)}$ Serologically, the first research linking schizophrenia and other psychoses to an increase in antibodies to T. gondii was published in 1953; 
since then, a number of studies have been carried out. ${ }^{7} \mathrm{~A}$ study of newborn and maternal sera of individuals who later developed schizophrenia reported more $T$. gondii antibodies in their sera compared to normal controls. ${ }^{8)}$ In addition, preliminary analysis of a cohort of individuals in the United States Military indicated that increased levels of Toxoplasma antibodies can be found in individuals prior to the onset of psychotic symptoms, thus obviating the possibility that the finding of increased levels of antibodies is an epiphenomenon associated with exposure occurring after the onset of schizophrenia. ${ }^{9)}$ Phee et al..${ }^{10)}$ using indirect fluorescent antibody technique (IFA), found higher positive reaction for $T$. gondii in chronic schizophrenia patients than in control group.

Chlamydiae were taxonomically categorized into their own order Chlamydiales, with one family, Chlamydiaceae, and a single genus, Chlamydia which included four species: Chlamydia (C.) trachomatis, C. pneumonia, $C$. psittaci, and C. pecorum. ${ }^{11)}$ Two of the species, $C$. trachomatis and $C$. pneumoniae are common human pathogens and can persist in infected monocytes. The primary targets of Chlamydophila infection in the brain are probably microglia cells, which arise from monocyte subpopulations. ${ }^{12)}$ Despite the presence of limited literature, it is evident that Chlamydia may be implicated in the pathogenesis of schizophrenia. Fellerhoff et al. ${ }^{13)}$ using n-polymerase chain reaction (PCR), found a significant prevalence of $C$. psittaci, C. pneumoniae, and C. trachomatis in schizophrenic patients, as compared to controls. Fellerhoff and Wank ${ }^{14)}$ also found that prevalence of Chlamydophila DNA in post-mortem brain frontal cortex from patients with schizophrenia was four times greater than in controls. Frykholm ${ }^{15)}$ suggested C. pneumonia as a common etiology of schizophrenia and multiple sclerosis, and reported improvement in several cases of psychotic patients by antibiotic therapy.

Considering all these findings, we conjectured that $T$. gondii and Chlamydia might play a key role in the etiology of schizophrenia. Although there exists several studies supporting association between schizophrenia and these infectious agents, to the best of our knowledge, almost no research dealing with the subject of present investigation was performed in Korea. In this study, we investigated whether there is a higher prevalence of T. gondii, C. trachomatis and $C$. pneumonia infection in schizophrenic patients. Likewise, we divided schizophrenic patients into seropositive and seronegative group based on seropositivity of immunoglobulin (Ig) and compared their features to figure out specific trait of infectious agent related to schizophrenic patients

\section{METHODS}

\section{Subjects}

Ninety-six patients with schizophrenia participated in the study. The number of female and male patients was 34 (35.4\%) and $62(64.6 \%)$, respectively. They were hospitalized or received out-patient services at the department of psychiatry of Soon Chun Hyang university hospital, Cheonan from July 1st, 2010 to June 30th, 2011. Clinical diagnoses were established according to Diagnostic and Statistical Manual-IV edition. As a structured tool, the Positive and Negative Syndrome Scale (PANSS) was used to assess the symptoms of the patients. Exclusion criteria included presence of a history of immunodeficiency disease, serious physical disease, neurological disease, or substance abuse. The illness duration in the schizophrenia patients was from 0.8 years to 50 years (mean, $13.76 \pm 11.15$ years), the age range was from 11 years to 61 years (mean, $46.14 \pm 13.15$ years).

Among the people who underwent physical examination for employment, 50 who had no history of physical, mental, or genetic disease were included in the study as the normal controls. They were matched with the schizophrenic patient group with respect to age and gender. The age range was from 25 years to 59 years (mean, 44.80 \pm 9.69 years). The number of female and male patients was $18(36 \%)$ and $32(64 \%)$, respectively. For all the subjects, written informed consent was obtained after the study procedure had been explained. The study protocols and the consent forms were approved by the Institutional Review Board of Soon Chun Hyang University (2010-40).

\section{Clinical Measures}

The patients were interviewed by a skilled psychiatrist by using Korean version of PANSS. PANSS is the most widely used measure of symptom severity in schizophrenia. The PANSS was developed by Kay et al. ${ }^{16)}$ (1987) and translated into Korean version by Kim, ${ }^{17)}$ and the reliability and validity were studied. PANSS is composed of 30 items, including 7 positive scales, 7 negative scales and 16 general psychopathology scales. The patient is rated from 1 to 7 on 30 different symptoms based on the interview as well as reports from family members or primary care hospital workers. This assessment requires approximately 30-40 minutes semi-structured interview. 


\section{Serum Collection \& Laboratory Assay}

Serum blood samples were obtained from the participants by venipuncture. The samples were stored at $4^{\circ} \mathrm{C}$ and tested for antibodies using enzyme-linked immunosorbent assay and IFA at Samkwang Medical Laboratories.

Serum toxoplasmosis antibody titer was evaluated by using chemiluminescent immunoassay (access $\operatorname{IgG}$ or IgM - TOXO, Beckman, Geneva, Switzerland). They are performed on microtiter plate reader (Access Immunoassay System, Sanofi Diagnostics Pasteur, Marnes-LaCoquette, France), which is an automated analyzer.

C. trachomatis IgG and IgM was assayed using EIA (BAG-C. trachomatis-EIA, BAG healthcare, Lich, Germany). Briefly, $100 \mu 1$ of diluted serum $(1: 101)$ was added into 96-microwell stripes coated with inactive antigen and incubated for 30 minutes at room temperature. After washing for four times with buffered wash solution, $100 \mu 1$ of peroxidase-conjugated antihuman antibody was added to the stripes and further incubated for 30 minutes at room temperature, with due protection from intense light. After washing for four times with buffered wash solution, $100 \mu 1$ of TMB substrate solution was added and incubated for 10 minutes at room temperature in the dark. After adding $100 \mu 1$ of stop solution, the absorbance was read using ELx $808^{\mathrm{TM}}$ Incubating Microplate Reader (BioTek, Lyon, France).

C. pneumonia $\operatorname{IgG}$ and IgM was assayed using IFA (CHLAMYDOPHILIA PNEUMONIAE IFA IgG or IgM, Vircell, Granada, Spain). All the assays were performed in duplicate using the manufacturer's recommended buffers, diluents, and substrates. The IFA test was performed by using prepared $C$. pneumoniae antigen with diluted serum
(1: 64) in PBS. In brief, $5 \mu 1$ of diluted serum, positive control serum and negative control serum was added into clean 30 -well slide and incubated for 30 minutes at $37^{\circ} \mathrm{C}$ in a humidified chamber. After three washes with PBS and soaking for 10 minutes, the slide was dried in air. Subsequently, $5 \mu 1$ of fluorescein isothiocyanate-labeled anti-human IgM or IgG diluted $1: 200$ in $0.001 \%$ Evans blue solution was added to each antigen spot and the slides were incubated for 30 minutes at $37^{\circ} \mathrm{C}$. Finally, they were washed as before, dried in air, and mounted in $50 \%$ glycerol-PBS (pH 8.6). The slides were examined with a $40 \times$ objective ( $\times 400$ magnification) using a fluorescence microscope.

\section{Statistical Analysis}

To analyze demographic data, two-tailed t-test was used for continuous covariates. For discrete covariates, chi-square test was used. The null hypothesis was rejected at $p<0.05$. All statistical analyses were performed with SPSS ver. 18.0 for Windows (SPSS Inc., Chicago, IL, USA).

\section{RESULTS}

\section{Bacterial and Protozoan Antibody Prevalence in Schizophrenic Patients and Controls}

We found that the group of schizophrenic patients had a significantly higher number of positive IgG antibody titers of $T$. gondii $(p=0.035)$ when compared to normal controls. In the group of Schizophrenic patients, significantly more positive $\operatorname{IgG}$ antibody titers against $C$. trachomatis $(p=0.015)$ was present when compared to healthy controls. No statistical difference could be meas-

Table 1. Comparison of class-specific reactivity to Toxoplasma (T.) gondii, Chlamydia (C.) pneumoniae and C. trachomatis agents in serum samples between schizophrenia patients and normal controls

\begin{tabular}{|c|c|c|c|c|c|c|}
\hline & \multicolumn{2}{|c|}{ T. gondii } & \multicolumn{2}{|c|}{ C. pneumoniae } & \multicolumn{2}{|c|}{ C. trachomatis } \\
\hline & Schizophrenia patients & Control group & Schizophrenia patients & Control group & Schizophrenia patients & Control group \\
\hline $\lg G+$ & $21(21.9)$ & $4(8.0)$ & $64(66.7)$ & $35(70.0)$ & $27(38.6)$ & $9(18)$ \\
\hline $\lg G-$ & $75(78.1)$ & $46(92)$ & $32(33.3)$ & $15(30.0)$ & $43(61.4)$ & $41(82)$ \\
\hline$\chi^{2}$ & \multicolumn{2}{|c|}{4.460} & \multicolumn{2}{|c|}{0.167} & \multicolumn{2}{|c|}{5.878} \\
\hline$p$ & \multicolumn{2}{|c|}{0.035} & \multicolumn{2}{|c|}{0.682} & \multicolumn{2}{|c|}{0.015} \\
\hline OR & \multicolumn{2}{|c|}{3.220} & \multicolumn{2}{|c|}{0.857} & \multicolumn{2}{|c|}{2.860} \\
\hline $95 \% \mathrm{Cl}$ & \multicolumn{2}{|c|}{$1.040-9.973$} & \multicolumn{2}{|c|}{$0.409-1.794$} & \multicolumn{2}{|c|}{$1.202-6.809$} \\
\hline $\operatorname{lgM}+$ & $0(0)$ & $1(2.0)$ & $14(14.6)$ & $4(8.0)$ & $7(10)$ & $7(14)$ \\
\hline $\operatorname{lgM}-$ & $96(100)$ & 49 (98) & $82(85.4)$ & $46(92.0)$ & $63(90)$ & $43(86)$ \\
\hline$\chi^{2}$ & \multicolumn{2}{|c|}{1.933} & \multicolumn{2}{|c|}{1.318} & \multicolumn{2}{|c|}{0.453} \\
\hline$p$ & \multicolumn{2}{|c|}{0.164} & \multicolumn{2}{|c|}{0.251} & \multicolumn{2}{|c|}{0.501} \\
\hline OR & & & \multicolumn{2}{|c|}{1.963} & \multicolumn{2}{|c|}{0.683} \\
\hline $95 \% \mathrm{Cl}$ & & & \multicolumn{2}{|c|}{$0.610-6.316$} & \multicolumn{2}{|c|}{$0.223-2.086$} \\
\hline
\end{tabular}

Ig, immunoglobulin; OR, odds ratio; $\mathrm{Cl}$, confidence interval. 
Table 2. Demographic variables of the schizophrenic patients, grouped by serum reactivity to IgG antibody of Toxoplasma (T.) gondii and Chlamydia (C.) trachomatis

\begin{tabular}{|c|c|c|c|c|c|c|c|c|}
\hline \multirow[b]{2}{*}{ Indexes } & \multicolumn{4}{|c|}{ T. gondii } & \multicolumn{4}{|c|}{ C. trachomatis } \\
\hline & $\begin{array}{l}\lg G+ \\
(n=21)\end{array}$ & $\begin{array}{l}\lg G- \\
(n=75)\end{array}$ & $\begin{array}{c}\text { Test } \\
\text { statistic }\end{array}$ & $p$ & $\begin{array}{l}\lg G+ \\
(n=27)\end{array}$ & $\begin{array}{l}\lg G- \\
(n=43)\end{array}$ & $\begin{array}{c}\text { Test } \\
\text { statistic }\end{array}$ & $p$ \\
\hline Gender & $17 \mathrm{M}, 4 \mathrm{~F}$ & $45 \mathrm{M}, 30 \mathrm{~F}$ & $3.149^{*}$ & 0.076 & $18 \mathrm{M}, 9 \mathrm{~F}$ & $31 \mathrm{M}, 12 \mathrm{~F}$ & $0.233^{*}$ & 0.630 \\
\hline Age, mean (SD) & $48.76(15.14)$ & $45.4(12.55)$ & $1.036^{\dagger}$ & 0.303 & 50.7 (12.39) & $50.14(9.91)$ & $0.210^{\dagger}$ & 0.834 \\
\hline Education (years), mean (SD) & $7.85(4.72)$ & $10.58(3.72)$ & $-2.745^{\dagger}$ & 0.007 & $9.9(4.81)$ & $9.43(4.09)$ & $0.428^{\dagger}$ & 0.670 \\
\hline Duration (years), mean (SD) & $13.33(10.89)$ & $13.89(11.30)$ & $-0.198^{\dagger}$ & 0.843 & $14.69(12.14)$ & $16.55(10.36)$ & $-0.664^{\dagger}$ & 0.509 \\
\hline Age at onset, mean (SD) & $34.90(15.45)$ & $31.28(11.57)$ & $1.147^{\dagger}$ & 0.254 & $35.58(13.50)$ & $33.58(12.64)$ & $0.612^{\dagger}$ & 0.543 \\
\hline $\begin{array}{l}\text { Number (\%) of patients with } \\
\text { family history of mental disorder }\end{array}$ & $5(23.8)$ & $5(6.7)$ & $5.167^{\star}$ & 0.023 & $1(3.7)$ & 7 (16.3) & $2.591^{*}$ & 0.107 \\
\hline
\end{tabular}

Ig, immunoglobulin; SD, standard deviation.

${ }^{*}$ Chi-squared test value; 'Student's t-test, t-value

Table 3. Score of Positive and Negative Syndrome Scale in schizophrenic patients, grouped by serum reactivity to IgG antibody of Toxoplasma (T.) gondii and Chlamydia (C.) trachomatis

\begin{tabular}{|c|c|c|c|c|c|c|c|c|}
\hline \multirow{2}{*}{$\begin{array}{l}\text { Positive and Negative } \\
\text { Syndrome Scale }\end{array}$} & \multicolumn{4}{|c|}{ T. gondii } & \multicolumn{4}{|c|}{ C. trachomatis } \\
\hline & $\begin{array}{l}\lg G+(n=21) \\
\text { mean }(S D)\end{array}$ & $\begin{array}{l}\lg G-(n=75) \\
\text { mean }(S D)\end{array}$ & t-value & $p$ & $\begin{array}{l}\lg G+(n=27) \\
\text { mean }(S D)\end{array}$ & $\begin{array}{l}\lg G-(n=43) \\
\text { mean }(S D)\end{array}$ & t-value & $p$ \\
\hline $\mathrm{Pl}$ & $3.24(1.76)$ & $3.6(1.57)$ & -0.91 & 0.365 & $3.15(1.46)$ & $2.88(1.30)$ & 0.79 & 0.431 \\
\hline P2 & $3.86(1.49)$ & $3.41(1.25)$ & 1.38 & 0.173 & $3.41(1.34)$ & $3.26(1.03)$ & 0.54 & 0.595 \\
\hline P3 & $2.19(1.5)$ & $2.72(1.74)$ & -1.27 & 0.209 & $2.15(1.41)$ & $2.16(1.33)$ & -0.04 & 0.965 \\
\hline P4 & $2.62(1.32)$ & $2.63(1.26)$ & -0.02 & 0.981 & $2.56(1.01)$ & $2.35(1.08)$ & 0.79 & 0.430 \\
\hline P5 & $1.95(1.66)$ & $2.04(1.46)$ & -0.24 & 0.814 & $1.85(1.35)$ & $1.53(1.08)$ & 1.09 & 0.308 \\
\hline P6 & $3.33(1.71)$ & $3.4(1.38)$ & -0.19 & 0.853 & $3.07(1.24)$ & $2.88(1.33)$ & 0.60 & 0.552 \\
\hline P7 & $2.43(1.21)$ & $2.63(1.35)$ & -0.61 & 0.546 & $2.37(1.15)$ & $2.35(1.04)$ & 0.08 & 0.936 \\
\hline Positive subscale score & $19.67(8.5)$ & $20.36(6.86)$ & -0.39 & 0.699 & $18.56(5.54)$ & $17.49(4.92)$ & 0.84 & 0.403 \\
\hline N1 & $3.76(1.04)$ & $3.09(1.32)$ & 2.14 & $0.035^{\star}$ & $3.30(1.17)$ & $3.37(0.95)$ & -0.30 & 0.768 \\
\hline N2 & $3.62(1.36)$ & $3.03(1.32)$ & 1.81 & 0.073 & $3.33(1.33)$ & $3.33(1.04)$ & 0.03 & 0.978 \\
\hline N3 & $3.62(1.36)$ & $3.29(1.38)$ & 0.96 & 0.341 & $2.96(1.37)$ & $3.49(1.12)$ & -1.75 & 0.085 \\
\hline N4 & $3.71(1.23)$ & $3.36(1.40)$ & 1.05 & 0.296 & $3.37(1.45)$ & $3.58(1.12)$ & -0.69 & 0.495 \\
\hline N5 & $3.71(1.31)$ & $3.35(1.41)$ & 1.07 & 0.286 & $3.52(1.42)$ & $3.49(1.12)$ & 0.10 & 0.922 \\
\hline N6 & $3.95(1.47)$ & $3.37(1.52)$ & 1.55 & 0.124 & $3.52(1.63)$ & $3.35(1.19)$ & 0.50 & 0.617 \\
\hline N7 & $2.57(1.33)$ & $1.97(1.15)$ & 2.04 & $0.045^{\star}$ & $1.93(1.04)$ & $1.98(0.96)$ & -0.21 & 0.835 \\
\hline Negative subscale score & $24.95(7.01)$ & $21.4(7.51)$ & 1.94 & 0.055 & $21.93(7.88)$ & $22.44(5.40)$ & -0.32 & 0.746 \\
\hline G1 & $2.57(1.63)$ & $2.21(1.34)$ & 1.03 & 0.305 & $2.00(1.18)$ & $2.23(1.36)$ & -0.73 & 0.466 \\
\hline G2 & $2.9(1.64)$ & $2.65(1.35)$ & 0.72 & 0.474 & $2.04(1.09)$ & $2.42(1.10)$ & -1.42 & 0.160 \\
\hline G3 & $1.67(1.80)$ & $1.67(1.30)$ & 0.00 & 1.00 & $1.19(0.48)$ & $1.26(0.62)$ & -0.50 & 0.617 \\
\hline G4 & $2.76(1.61)$ & $2.48(1.37)$ & 0.80 & 0.425 & $2.15(1.17)$ & $2.37(1.11)$ & -0.80 & 0.424 \\
\hline G5 & $1.95(1.20)$ & $1.68(1.08)$ & 1.00 & 0.322 & $1.59(1.19)$ & $1.70(0.83)$ & -0.44 & 0.664 \\
\hline G6 & $2.52(1.37)$ & $2.27(1.27)$ & 0.81 & 0.421 & $1.85(1.10)$ & $2.35(1.13)$ & -1.81 & 0.075 \\
\hline G7 & $3.14(1.11)$ & $2.43(1.15)$ & 2.54 & 0.013 & $2.56(1.09)$ & $2.65(1.02)$ & -0.37 & 0.711 \\
\hline G8 & $2.48(1.29)$ & $2.6(1.38)$ & -0.37 & 0.713 & $2.44(1.31)$ & $2.4(1.14)$ & 0.17 & 0.869 \\
\hline G9 & $2.62(1.63)$ & $3.17(1.52)$ & -1.46 & 0.149 & $2.7(1.24)$ & $2.47(1.30)$ & 0.76 & 0.448 \\
\hline G10 & $2.33(1.39)$ & $1.83(1.17)$ & 1.69 & 0.095 & $2.44(1.25)$ & $1.81(1.14)$ & 2.17 & $0.033^{*}$ \\
\hline G11 & $2.67(1.24)$ & $2.33(1.19)$ & 1.13 & 0.263 & $2.37(1.18)$ & $2.30(0.96)$ & 0.26 & 0.793 \\
\hline G12 & $3.67(1.28)$ & $4.05(1.27)$ & -1.23 & 0.222 & $4.11(1.25)$ & $3.81(1.01)$ & 1.09 & 0.278 \\
\hline G13 & $3.48(1.17)$ & $2.79(1.32)$ & 2.17 & $0.033^{*}$ & $3.11(1.28)$ & $2.91(1.00)$ & 0.75 & 0.458 \\
\hline G14 & $2.43(1.21)$ & $2.31(1.37)$ & 0.37 & 0.712 & $2.11(1.16)$ & $2.26(1.12)$ & -0.52 & 0.604 \\
\hline G15 & $1.81(1.25)$ & $1.95(1.27)$ & -0.44 & 0.662 & $1.59(0.93)$ & $1.42(0.63)$ & 0.94 & 0.396 \\
\hline G16 & $3.14(1.24)$ & $2.85(1.33)$ & 0.89 & 0.374 & $2.59(1.34)$ & $2.77(0.87)$ & -0.66 & 0.549 \\
\hline $\begin{array}{l}\text { General psychopathology } \\
\text { subscale score }\end{array}$ & $40.95(15.92)$ & $39.53(10.52)$ & 0.48 & 0.63 & $36.81(10.51)$ & $36.58(6.73)$ & 0.11 & 0.910 \\
\hline
\end{tabular}

Ig, immunoglobulin; SD, standard deviation.

${ }^{*} p<0.05$ by independent t-test. 
ured for C. pneumoniae. The rates of IgM antibodies of the schizophrenia patients were not significantly higher than that of the control group. Using the exposure level of the control groups as reference, the odds ratio of schizophrenia associated with $T$. gondii and $C$. trachomatis infection was 3.22 and 2.86, respectively (Table 1).

\section{Comparing Demographic Variables in Groups Divided with Seropositivity in Schizophrenia}

The schizophrenic patients were separated into two groups on the basis of presence of detectable IgG antibody to infectious agents (IgG + group and $\mathrm{IgG}-$ group). For T. gondii, $\mathrm{IgG}+$ group showed significantly less years of education ( $7.85 \pm 4.72$ years) when compared to $\mathrm{IgG}-$ group $(10.58 \pm 3.72$ years; $p=0.007)$. Furthermore, IgG + group had significantly higher percentage of familial history of mental illness $(23.8 \%)$ when compared to IgGgroup $(6.7 \%)$. Nevertheless, there was no significant difference in terms of gender, age, duration of illness, and age of onset of schizophrenia. C. trachomatis showed no significant differences between $\mathrm{IgG}+$ group and $\mathrm{IgG}-$ group with respect to gender, age, education, duration of illness, age of onset of schizophrenia and number of patients with family history of mental disorder (Table 2).

\section{Comparing PANSS Subscale Score of IgG+ and IgG- Group}

The PANSS subscale scores of the two groups are shown in Table 3. For $T$. gondii, the score of N1 (blunted affect), N7 (stereotyped thinking), G13 (Disturbance of volition) subscale was significantly higher in $\mathrm{IgG}+$ group when compared to IgG group. For C. trachomatis, the score of G10 (disorientation) subscale was significantly higher in $\mathrm{IgG}+$ group when compared to $\mathrm{IgG}-$ group.

\section{DISCUSSION}

In the present study, we investigated the rate of positive serological reaction to $T$. gondii, $C$. pneumoniae, and $C$. trachomatis in 96 schizophrenic patients and 50 normal controls. Likewise, we divided schizophrenic patients into seropositive and seronegative group based on seropositivity of Ig and compared their features to figure out specific trait of infectious agent-related schizophrenic patients.

We found that the schizophrenic patients had a significantly higher rate of positive IgG antibodies to infectious agents as compared to the normal controls. Especially, antibodies to $T$. gondii and C. trachomatis were overrepresented in schizophrenic patients. This finding suggests that $T$. gondii and $C$. trachomatis infections are associated with schizophrenia.

In accordance to the present study, some of the previous studies reported that Toxoplasma infection represents one risk factor for schizophrenia. Wang et al. ${ }^{3)}$ reported an association between Toxoplasma infection and first episode of schizophrenia, suggesting a 2-5 times increased risk of schizophrenia due to chronic Toxoplasma infection. Niebuhr et al. ${ }^{9)}$ using samples obtained from United States Military members prior to schizophrenia diagnosis, found significant positive association between $T$. gondii IgG antibody and schizophrenia. Yolken et al. ${ }^{18)}$ found demonstrated that individuals with first episode schizophrenia had significantly increased levels of $\operatorname{IgG}, \operatorname{IgM}$ and IgA class antibodies to T. gondii. Phee et al. ${ }^{10)}$ found higher positive reaction for $T$. gondii in chronic schizophrenia patients than control group, especially patients with negative symptoms and our findings are consistent with these studies. However, there are a number of other studies, which did not correspond to the results of present study. Krause et al. ${ }^{19)}$ found no statistical differences measured for $T$. gondii. Further research is needed to clarify the inconsistency in the study.

For $C$. trachomatis, our findings were in accordance with previous studies that concluded that $C$. trachomatis infection is associated with schizophrenia. Krause et al. ${ }^{19)}$ found significantly higher rates of positive antibodies to C. trachomatis when compared to the other infectious agents, suggesting infection as one of the contributing factors for schizophrenia amongst others, like genetic disposition. Fellerhoff et al. ${ }^{13)}$ found significant prevalence of chlamydial infection in schizophrenic group, suggesting chronic infections with chlamydiaceae as the main pathogenic factors in the pathogenesis of schizophrenia, and the requirement of immunotherapy to restore the balance of immune subpopulations. To the best of our knowledge, this is the first report on a positive association of $C$. trachomatis with schizophrenia in a Korean population.

In the case of $C$. pneumoniae, despite the presence of several previous studies supporting the contention that $C$. pneumoniae is a possible risk factor for schizophrenia, ${ }^{13,14)}$ no statistical difference could be measured. Nevertheless, a research corresponded to the result of our study but the reason was uncertain. ${ }^{19)}$ In our study, schizophrenia and control group both showed high IgG seropositivity. High prevalence of $C$. pneumoniae in Korea and the character of their environment could be the leading causes for high seropositivity in both schizophrenia and control group. 
Korea is highly endemic with C. pneumoniae infection, with increased prevalence in adults. A research has demonstrated that in healthy subjects over 21 years of age, $C$. pneumoniae IgG antibody was present in $70 \%$ of males and $56 \%$ of females. ${ }^{20)}$

In the present study, it was observed that mainly IgG and not IgM antibodies were elevated in condition of schizophrenia, and this could further indicate that the infections are not acute any more, but have progressed to dormant infections with a persistent immune response. As IgG antibodies are involved in secondary immune response, IgM antibodies appear early in the course of an infection and usually reappear. ${ }^{21)}$ In our study, we just showed the rates of antibody titers, the infectious agents were not investigated directly with PCR. However, there is no evidence from this study that patients with schizophrenia have an increased prevalence of acute infections, but clearly, these patients have had more infections in the past and/or are suffering from a chronic infectious condition.

We also compared the characteristics between schizophrenia patients with detectable IgG antibody (the positive group) and those without detectable $\operatorname{IgG}$ antibody (the negative group) to $T$. gondii and $C$. trachomatis. For T. gondii, positive group showed less years of education. We cannot assert that infection is the only factor related to years of education, but our findings might be related to previous studies, which demonstrated that schizophrenic patients, who had gestational exposure to infectious agents showed executive dysfunction when compared to unexposed group. ${ }^{22)}$ Impaired executive function might have influenced the ability to keep up their learning, eventually making them to be dropped out of school. In addition, positive group showed higher rate of familial history of mental disorder. This could be a supportive finding for genetic susceptibility of those people, who became schizophrenic due to infection. We conjectured that if high rate of familial history of mental disorder indicates inherited genetic fragility, higher rate of familial history would be present in sero-positive group, which has more probability for infection as etiology of their disorder, when compared to sero-negative group. A number of studies have shown evidence on the interaction of predisposing genes with one or more infectious agents to cause schizophrenia. ${ }^{7,23)}$ C. trachomatis showed no significant difference in character between seropositive and seronegative group.

In addition, we have also compared the PANSS subscale score between schizophrenic patients with seropositive and seronegative group for T. gondii and C. tra- chomatis infection. For $T$. gondii, the seropositive group scored significantly higher score in several negative subscales and one general psychopathology scale, 'disturbance of volition'. Fellerhoff et al., ${ }^{13)}$ treated patients with schizophrenia and other mental disorders with evidence of infection by using adoptive immunotherapy and antibiotics, but no antipsychotics. The improvement in the status of the patients was mainly related with regain of energy and volition, improvement of social withdrawal, which are main components of negative symptom. Based on these findings, we cautiously suggest the relation between negative symptoms and 'disturbance of volition' with infectious agent. In the case of $C$. trachomatis infection, the seropositive group scored significantly higher score in one general psychopathology subscale, disorientation. Still, evidence to underpin relation between infectious agents and disorientation is insufficient.

Despite numerous studies performed to reveal relation between infectious agents and schizophrenia, it is still controversial whether infectious agents play a causal role in inducing psychotic symptoms. Proving causality is one of the major limitations of studies about the association of schizophrenia and infections. As schizophrenia is constituted of a number of inhomogeneous symptoms, it seems more probable that infections might just be one of the contributing factors among others. ${ }^{13)}$ In the case of schizophrenia, one possible causality could be that infectious agents do not directly cause psychiatric symptoms, but influence the immune balance via the status of a chronic infection. We assume that these different infections have an impact on the immune system and therefore might contribute to psychiatric symptoms. Several studies have identified the important role of immunological parameters in schizophrenia. ${ }^{23)}$ Recently, it has been demonstrated that proinflammatory cytokines that could enhance the activity of the enzyme, indoleamine 2,3-dioxygenase (IDO) plays an important role in the pathophysiology of schizophrenia. The IDO increases tryptophan degradation into kynurenine and decreases tryptophan availability in the brain to synthesize neurotransmitters. ${ }^{24)}$ Reduced type-1 immune response and Increased type- 2 immune response in schizophrenic patients promote the production of the endogenous $\mathrm{N}$ Methyl-D-aspratate (NMDA) receptor antagonist, kynurenic acid. A hypofunction of the glutamatergic cortico-striatal pathway is associated with opening of the thalamic filter, which leads to an uncontrolled flow of sensory information to the cortex and promotes psychotic symptoms. ${ }^{25}$

There are some limitations in our study: First, the sam- 
ple size was rather small and therefore the statistical power might be restricted. Although there were 96 patients with schizophrenia in the study, the number of the seropositive patients for T. gondii $(\mathrm{n}=21), C$. pneumoniae $(\mathrm{n}=64)$, C. trachomatis $(\mathrm{n}=27)$ were small. Therefore, the results of the clinical symptoms of the seropositive and seronegative schizophrenia need to be further verified, especially through prospective studies. Second, it was not possible to evaluate the rate of sexual activity in patients and controls. This aspect seems to be important, as C. trachomatis is a sexually transmitted disease. There are studies showing that schizophrenic patients suffer from sexual dysfunction and possess less social activity and have therefore an impaired sexual life. ${ }^{26)}$ It also remains possible that schizophrenic patients might show riskier sexual behavior.

In conclusion, the present research found that the prevalence of IgG antibody to $T$. gondii and $C$. trachomatis in the patients with schizophrenia was higher than that of the control groups. These findings suggest that the elevated rate of infectious agents within the schizophrenic patients could provoke an immunological disturbance that might influence the cerebral neurotransmitter balance. This study lent further weight to the hypothesis that exposure to T. gondii and C. trachomatis may be the risk factors for schizophrenia. Further studies investigating the association between the infection status and immune parameters are needed.

\section{REFERENCES}

1. Brown AS. Prenatal infection as a risk factor for schizophrenia. Schizophr Bull 2006;32:200-202.

2. Fuller Torrey E, Yolken RH. Familial and genetic mechanisms in schizophrenia. Brain Res Brain Res Rev 2000;31: 113-117.

3. Wang HL, Wang GH, Li QY, Shu C, Jiang MS, Guo Y. Prevalence of Toxoplasma infection in first-episode schizophrenia and comparison between Toxoplasma-seropositive and Toxoplasma-seronegative schizophrenia. Acta Psychiatr Scand 2006;114:40-48.

4. Torrey EF, Bartko JJ, Lun ZR, Yolken RH. Antibodies to Toxoplasma gondii in patients with schizophrenia: a metaanalysis. Schizophr Bull 2007;33:729-736.

5. Montoya JG, Liesenfeld O. Toxoplasmosis. Lancet 2004; 363:1965-1976.

6. Estran C, Brenier-Pinchart MP, Pelletier L, Cesbron-Delauw MF, Pelloux H. Toxoplasma gondii in human astrocytes in vitro: interleukin (IL)-12 and IL-10 do not influence cystogenesis. J Parasitol 2006;92:1108-1110.

7. Yolken RH, Torrey EF. Are some cases of psychosis caused by microbial agents? A review of the evidence. Mol Psychiatry 2008; 13:470-479.

8. Brown AS, Schaefer CA, Quesenberry CP Jr, Liu L, Babulas VP, Susser ES. Maternal exposure to toxoplasmosis and risk of schizophrenia in adult offspring. Am J Psychiatry 2005; 162:767-773.

9. Niebuhr DW, Millikan AM, Cowan DN, Yolken R, Li Y,
Weber NS. Selected infectious agents and risk of schizophrenia among U.S. Military personnel. Am J Psychiatry 2008; 165:99-106.

10. Phee SS, Park H, Park MC. Antibody titers of Toxoplasma gondii among patients with mental disorders. $J$ Wonkwang Med Sci 1999;15:33-42.

11. Everett KD, Bush RM, Andersen AA. Emended description of the order Chlamydiales, proposal of Parachlamydiaceae fam. nov. and Simkaniaceae fam. nov., each containing one monotypic genus, revised taxonomy of the family Chlamydiaceae, including a new genus and five new species, and standards for the identification of organisms. Int $J$ Syst Bacteriol 1999;49 Pt 2:415-440.

12. Kaur C, Dheen ST, Ling EA. From blood to brain: amoeboid microglial cell, a nascent macrophage and its functions in developing brain. Acta Pharmacol Sin 2007;28:10871096.

13. Fellerhoff B, Laumbacher B, Wank R. High risk of schizophrenia and other mental disorders associated with chlamydial infections: hypothesis to combine drug treatment and adoptive immunotherapy. Med Hypotheses 2005;65:243-252.

14. Fellerhoff B, Wank R. Increased prevalence of Chlamydophila DNA in post-mortem brain frontal cortex from patients with schizophrenia. Schizophr Res 2011;129:191195.

15. Frykholm BO. On the question of infectious aetiologies for multiple sclerosis, schizophrenia and the chronic fatigue syndrome and their treatment with antibiotics. Med Hypotheses 2009;72:736-739.

16. Kay SR, Fiszbein A, Opler LA. The positive and negative syndrome scale (PANSS) for schizophrenia. Schizophr Bull 1987:13:261-276.

17. Kim CY. Psychiatric assessment instruments. 1st ed. Seoul: Hana Medical Press;2001. p.61-62.

18. Yolken RH, Bachmann S, Ruslanova I, Lillehoj E, Ford G, Torrey EF, et al. Antibodies to Toxoplasma gondii in individuals with first-episode schizophrenia. Clin Infect Dis 2001;32:842-844.

19. Krause D, Matz J, Weidinger E, Wagner J, Wildenauer A, Obermeier $\mathrm{M}$, et al. The association of infectious agents and schizophrenia. World J Biol Psychiatry 2010;11:739-743.

20. Choi TY, Kim DA, Kim SK, Kang JO, Park SS, Jung SR. Prevalence of specific antibodies to Chlamydia pneumoniae in Korea. J Clin Microbiol 1998;36:3426-3428.

21. Haynes BF, Fauci AS. Introduction to the immune system. In: Fauci AS, Braunwald E, Kasper DL, Hauser SL, Longo $D L$, Jameson $J L$, et al., editors. Harrison's principles of internal medicine. 16th ed. New York:McGraw Hill;2005. p.1907-1930.

22. Brown AS, Vinogradov S, Kremen WS, Poole JH, Deicken $\mathrm{RF}$, Penner JD, et al. Prenatal exposure to maternal infection and executive dysfunction in adult schizophrenia. Am J Psychiatry 2009;166:683-690.

23. Miüller N, Schwarz MJ. The immunological basis of glutamatergic disturbance in schizophrenia: towards an integrated view. J Neural Transm Suppl 2007;(72):269-280.

24. Hariri AG, Karadag F, Gurol DT, Aksoy UM, Tezcan AE. Sexual problems in a sample of the Turkish psychiatric population. Compr Psychiatry 2009;50:353-360.

25. Myint AM, Schwarz MJ, Steinbusch HW, Leonard BE. Neuropsychiatric disorders related to interferon and interleukins treatment. Metab Brain Dis 2009;24:55-68.

26. Carlsson A. The neurochemical circuitry of schizophrenia. Pharmacopsychiatry 2006;39(Suppl 1):S10-S14. 